\title{
Correlação clínica entre estrutura e função no glaucoma: classificação estrutural de Armaly e o sistema de estadiamento funcional de Brusini
}

\author{
Clinical correlation between structural and functional assessment in \\ glaucoma: Armaly cup to disk ratio and Brusini glaucoma staging system
}

\author{
Adriana Chaves de Oliveira ${ }^{1}$ \\ Flávia Chaves de Oliveira ${ }^{2}$ \\ Marcos Bottene Villa Albers ${ }^{3}$ \\ Ralph Cohen ${ }^{4}$ \\ Niro Kasahara $^{5}$
}

Trabalho realizado na Faculdade de Ciências Médicas da Santa Casa de São Paulo - FCMSCSP - São Paulo (SP) - Brasil.

${ }^{1}$ Especializanda em Glaucoma do Setor de Glaucoma da Faculdade de Ciências Médicas da Santa Casa de São Paulo - FCMSCSP - São Paulo (SP) - Brasil.

${ }^{2}$ Especializanda em Órbita e Oculo-plástica do Setor de Glaucoma da FCMSCSP - São Paulo (SP) - Brasil.

${ }^{3}$ Residente do segundo ano do Departamento de Oftalmologia da FCMSCSP - São Paulo (SP) - Brasil.

${ }^{4}$ Chefe do Setor de Glaucoma da FCMSCSP - São Paulo (SP) - Brasil.

${ }^{5}$ Assistente do Setor de Glaucoma da FCMSCSP - São Paulo (SP) - Brasil.

Endereço para correspondência: Adriana Chaves de Oliveira. Rua General Jardim, 630 - Apto. 82 - São Paulo (SP) CEP 01223-010

E-mail: drychaves@ig.com.br

Recebido para publicação em 21.08.2006

Última versão recebida em 12.09.2007

Aprovação em 09.11.2007

Nota Editorial: Depois de concluída a análise do artigo sob sigilo editorial e com a anuência do Dr. Riuitiro Yamane sobre a divulgação de seu nome como revisor, agradecemos sua participação neste processo.

\begin{tabular}{|c|}
\hline RESUMO \\
\hline Objetivo: Avaliar a correlação entre a análise estrutural de Armaly e o \\
estadiamento funcional de Brusini em pacientes glaucomatosos. Métodos: \\
Setenta e oito olhos de 42 pacientes com glaucoma de ângulo aberto \\
realizaram exame de campo visual com o campímetro Humphrey Field \\
Analyzer II (programa SITA 24-2) e avaliação do disco óptico com lente \\
Volk 78D por dois examinadores independentes. O disco óptico foi clas- \\
sificado de acordo com a relação escavação/disco (C/D), de 0,1 a 1,0. Os \\
valores do "Mean Deviation" (MD) e "Pattern Standard Deviation" (PSD) \\
de cada olho foram inseridos na avaliação de estadiamento de Brusini, para \\
estabelecer um valor de gravidade do glaucoma. A relação escavação/ \\
disco e os valores de Brusini foram colocados em diagrama para serem \\
correlacionados (coeficiente de correlação de Spearman, rho). Os valores \\
de Armaly foram transformados em escala logarítmica e os dados foram \\
inseridos em um diagrama com os valores de Brusini para cada paciente. \\
Resultados: As análises de Armaly e Brusini apresentaram baixa correla- \\
ção(rho=0,340). A correlação, não-logarítmica elogarítmica, entre Armaly \\
e Brusini mostrou-se lineare baixa. Conclusão: Apesar dabaixa correlação, \\
a avaliação estrutural de Armaly e a análise funcional do estadiamento de \\
Brusini proporcionaram medidas não independentes do glaucoma. \\
\hline
\end{tabular}

Descritores: Glaucoma/classificação; Glaucoma/diagnóstico; Campo visual; Perimetria/ métodos

\section{INTRODUÇÃO}

O glaucoma primário de ângulo aberto é uma neuropatia óptica crônica progressiva que tem como principal fator de risco o aumento da pressão intra-ocular e é caracterizado por alterações típicas do disco óptico e da camada de fibras nervosas da retina ${ }^{(1)}$. O glaucoma possui vários métodos para avaliação e acompanhamento de sua evolução, entre eles a classificação estrutural de Armaly, o sistema de estadiamento funcional de Brusini, a biomicroscopia de fundo de olho e a perimetria computadorizada.

Na avaliação da gravidade do glaucoma, são utilizados parâmetros estruturais derivados da observação do disco óptico e da camada de fibras nervosas da retina, bem como parâmetros funcionais derivados da perimetria computadorizada.

A relação entre as avaliações estrutural e funcional no glaucoma é controversa. Muitos estudos definiram uma relação curvilínea ou não-linear 
entre o número de células ganglionares da retina e a sensibilidade retínica medida em decibéis $(\mathrm{dB})^{(2-6)}$. Porém, os parâmetros estruturais do disco óptico foram relacionados com a função retínica, utilizando escala não logarítmica, e foi observado uma relação linear entre as duas funções ${ }^{(7)}$.

Alguns autores acreditam que as alterações estruturais do disco óptico e da camada de fibras nervosas da retina e as alterações funcionais fornecem informações independentes e utilizam o termo progressão estrutural e progressão funcional do glaucoma, ao contrário de simplesmente dizer progressão da doença ${ }^{(8-10)}$.

A avaliação da gravidade da doença pela classificação estrutural de Armaly consiste na verificação da relação entre o diâmetro da escavação do disco óptico e de seu diâmetro propriamente dito, medida em décimos, e varia de 0,1 (escavação mínima) até 1,0 (escavação total). É uma avaliação subjetiva, com grande variabilidade entre diferentes observadores, porém é a mais utilizada na prática diária.

A escala de estadiamento do glaucoma proposta por Brusini correlaciona a perda geral de campo visual, "mean deviation" (MD), com a representação numérica da forma de ilha de visão do paciente em relação à população normal, "corrected pattern standard deviation" (CPSD) ou "pattern standard deviation" (PSD), e classifica o glaucoma em cinco estádios, sendo o 1 o mais leve e o 5 o mais grave ${ }^{(11-12)}$.

O objetivo deste estudo foi correlacionar clinicamente os parâmetros estruturais do glaucoma, definidos por Armaly, com a avaliação funcional, proposta por Brusini e verificar se esta correlação respeita uma curva de função linear ou não.

\section{MÉTODOS}

Este estudo foi aprovado pelo comitê de ética em pesquisa da Irmandade da Santa Casa de Misericórdia da São Paulo (protocolo número 278/05) e realizado entre junho e julho de 2005.

$\mathrm{Na}$ análise gráfica, a correlação entre a avaliação estrutural de Armaly e a análise funcional de Brusini foi colocada em escala logarítmica, para ser melhor observada.

\section{Amostra e critérios de seleção}

Foi realizado estudo transversal, selecionando 80 olhos de 42 pacientes com glaucoma primário de ângulo aberto (GPAA) do setor de Glaucoma do Departamento de Oftalmologia da Santa Casa de São Paulo, que preenchiam os seguintes critérios de inclusão: qualquer sexo e idade, acuidade visual maior que 20/40 com melhor correção óptica, sem catarata ou doenças da córnea ou da retina, não ter sido submetido a procedimentos cirúrgicos oftálmicos anteriores e ter pelo menos uma perimetria computadorizada com índices confiáveis (menos de 33\% de falso positivo e falso negativo e menos de $20 \%$ de perda de fixação). Pacientes com outros tipos de glaucoma, que não o primário de ângulo aberto, foram excluídos do estudo.

O glaucoma primário de ângulo aberto foi definido como a presença de defeito localizado ("notch") ou difuso da rima neural e/ou presença de defeito característico na perimetria computadorizada, a saber, três ou mais pontos com $\mathrm{p}<5 \%$ no gráfico "pattern deviation" (PD), PSD com $\mathrm{p}<5 \%$ ou GHT fora dos limites normais, em pacientes com seio camerular aberto à gonioscopia, independente do valor da pressão intra-ocular (PIO).

A amostra foi formada em sua maioria por pacientes de cor parda (18) e uma minoria de cor branca (11) e preta (13). Foram avaliados 35 pacientes do sexo feminino e 7 pacientes do sexo masculino, ou seja, uma proporção aproximada de seis mulheres para cada homem. A média de idade dos pacientes foi de $60,26 \pm 9,9650$ anos.

\section{Procedimentos}

Todos os pacientes foram submetidos a exame oftalmológico completo, incluindo medida da acuidade visual com melhor correção óptica; biomicroscopia de segmento anterior, avaliando alterações corneais e da câmara anterior; biomicroscopia de fundo de olho sob midríase medicamentosa, para avaliação do disco óptico com lente de Volk de 78 dioptrias, que produz imagem ampliada e invertida do disco óptico com iluminação da lâmpada de fenda. O paciente foi examinado por dois examinadores independentes, que avaliaram a relação escavação/cúpula (disco) de acordo com a classificação de Armaly ${ }^{(13)}$.

Os pacientes também foram submetidos ao exame de campo visual, mediante perimetria computadorizada com o perímetro Humphrey Field Analyzer II (modelo 750, Zeiss-Humphrey Systems, San Leandro-CA, EUA), programa SITA 24-2, com defeito refrativo corrigido e o exame supervisionado por um técnico após instruções. Este é um teste psicofísico, que avalia o campo visual por alterações neurológicas. O paciente recebe estímulo branco, projetado em fundo branco e é orientado a responder apertando um botão quando o estímulo é percebido. A confiabilidade do exame depende da cooperação do paciente.

\section{Análise dos resultados e estatística}

As medidas estruturais da escala de Armaly, usadas pelos dois examinadores foram comparadas com estatística Kappa, que avalia o nível de concordância entre observadores independentes.

Os valores de MD e PSD dos exames perimétricos foram inseridos na escala de Brusini para determinar o estádio da doença para cada olho.

Os dados estruturais e as medidas funcionais de cada paciente foram inseridos no gráfico para avaliar a correlação entre ambos.

As medidas estruturais foram colocadas no eixo das abscissas (x) e as medidas funcionais no das ordenadas (y). Uma curva foi derivada a partir do gráfico, bem como sua equação para determinar o valor do coeficiente de correlação (r). A correlação entre as duas funções foi avaliada de acordo com o coeficiente de correlação de Spearman (rho).

Os dados estruturais de Armaly foram convertidos para escala logarítmica e correlacionados com os dados da avalia- 
ção funcional de Brusini. A correlação entre as duas funções foi avaliada pelo coeficiente de correlação.

\section{RESULTADOS}

A análise da concordância entre os dois observadores da avaliação estrutural de acordo com a escala de Armaly revelou quase perfeita concordância entre ambos $(\mathrm{k}=0,924)$.

A figura 1 mostra a correlação entre a avaliação estrutural de Armaly e a análise funcional de Brusini. A correlação foi linear e o coeficiente de correlação (r) resultou em 0,337 (intervalo de confiança de $95 \%$ de 0,1223 a 0,5216 e p=0,0027), revelando baixa correlação.

O grau de correlação entre as duas variáveis avaliadas pelo coeficiente de correlação de Spearman resultou rho $=0,340$ (intervalo de confiança de $95 \%$ de 0,126 a $0,524, p=0,003$ ).

A figura 2 mostra a correlação entre a análise funcional de Brusini e a avaliação estrutural de Armaly em escala logarítmica. A correlação permaneceu linear e o coeficiente de correlação resultou em 0,2697 (intervalo de confiança de $95 \%$ de 0,0486 a 0,4656 e p=0,0177), também revelando baixa correlação.

\section{COMENTÁRIOS}

Nosso estudo revelou correlação linear entre a medida de função visual e estrutura do nervo óptico, diferindo da maioria dos estudos da literatura.

As diferenças nos métodos de análise comparativa das funções podem explicar essa diferença. Alguns autores consideraram a relação entre a área da rima neural e o dano funcional em decibéis medido pelo perímetro Octopus em pacientes normais, suspeitos e glaucomatosos ${ }^{(2)}$. O defeito do campo visual em decibéis medido pelo Octopus foi correlacionado à área da rima neural em 410 pacientes glaucomatosos ${ }^{(3)}$. Outros autores correlacionaram o defeito do campo visual em decibéis medido pelo perímetro Humphrey e a área da rima neural medida pela tomografia de varredura a laser em pacientes normais e glaucomatosos $^{(4)}$. A perda relativa da área da rima neural medida pela tomografia de varredura a laser, também, foi correlacionada com o defeito funcional em decibéis em pacientes glaucomatosos, hipertensos oculares e normais ${ }^{(5)}$. Outra correlação observada, por diversos especialistas, foi entre a sensibilidade média do campo visual medida em decibéis pelo Humphrey e a medida da espessura da camada de fibras nervosas da retina em pacientes normais e glaucomatosos ${ }^{(6,9)}$. Todos esses autores observaram relação não linear (exponencial ou logarítmica) entre as medidas estruturais relacionadas ao número de células ganglionares da retina e a sensibilidade retínica medida em decibéis.

Neste estudo, utilizamos uma medida funcional e outra estrutural. Não utilizamos a área da rima ou da camada de fibras nervosas da retina, mas uma medida clínica, a saber: avaliação estrutural de Armaly que compara o tamanho da escavação com o tamanho do disco óptico que embora pouco precisa e com

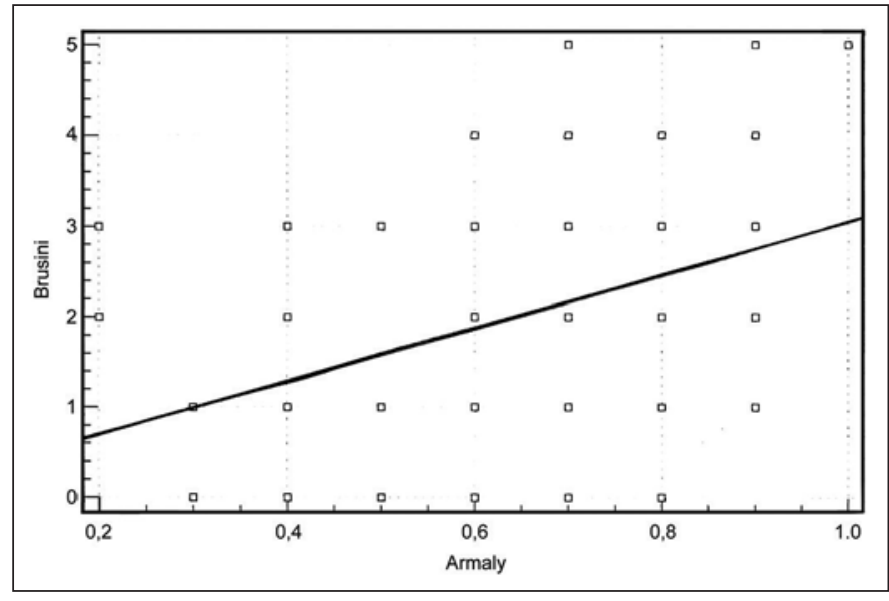

Figura 1 - Correlação entre a avaliação estrutural de Armaly e a análise funcional de Brusini em pacientes com glaucoma de ângulo aberto da Santa Casa de São Paulo, 2005

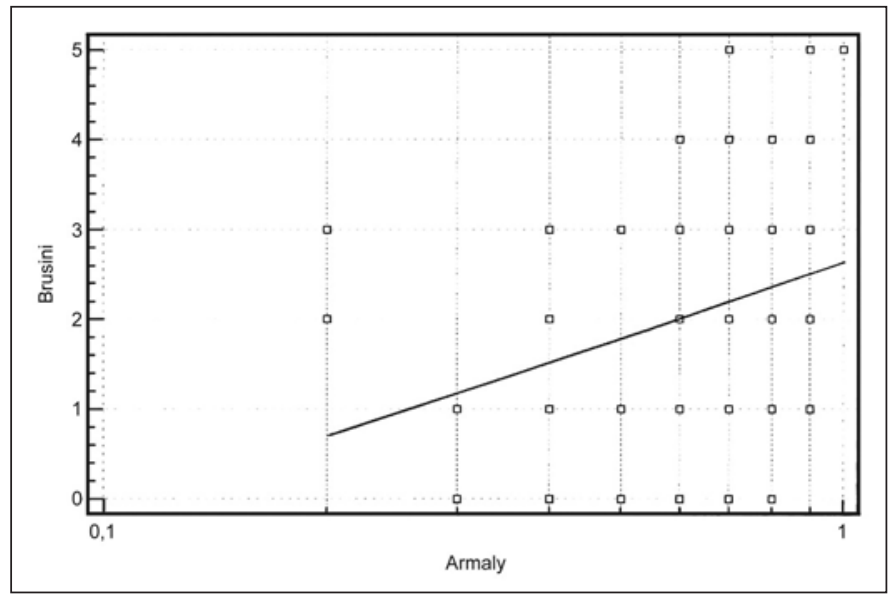

Figura 2 - Correlação entre análise funcional de Brusini e a avaliação estrutural de Armaly em escala logarítmica em pacientes com glaucoma de ângulo aberto da Santa Casa de São Paulo, 2005

grande variabilidade, tanto intra quanto entre observadores, é a mais utilizada na prática clínica. Para avaliação da função visual, utilizamos a escala de Brusini, que correlaciona o valor do "mean deviation" (índice que expressa uma medida geral da função visual) com o valor do "pattern standard deviation" (índice que evidencia os defeitos localizados do campo visual). A utilidade desta escala é comprovada por diferentes estudos ${ }^{(11-12)}$.

Ao transformarmos a escala de Armaly numa função logarítmica e correlacioná-la com a escala de Brusini, que é uma função logarítmica, não observamos diferença na correlação entre ambas. As funções continuaram a apresentar fraca correlação, porém mantendo relação linear e concordando com a literatura. Houveram autores que ao transformarem os valores da sensibilidade retínica numa escala não logarítmica, observaram relação linear entre a estrutura e a função ${ }^{(6,9)}$. Outros autores, comentando o modelo para análise da amplitude do potencial visual evocado multifocal com a perda das células 
ganglionares da retina, declararam que existe relação linear entre a perda de campo visual medida em escala anti-logarítmica e a perda de células ganglionares ${ }^{(7)}$. As diferenças entre os métodos de estudo podem justificar essas diferenças.

A correlação entre a escala funcional de Brusini e a escala estrutural de Armaly revelou-se baixa neste estudo ( $r$ ho $=0,340$ ). Este resultado nos leva a concluir que a escala de Armaly e a de Brusini nos fornecem medidas não independentes do glaucoma, nesta amostra.

A análise estrutural inclui a área da rima neural e o volume da escavação, que podem ser obtidos com medidas morfométricas usando métodos de imagem para avaliar o disco óptico ou a espessura da camada de fibras nervosas da retina ${ }^{(8)}$. Muitas dessas medidas não representam ou não são de fato parâmetros estruturais reais do glaucoma, mas substitutos da perda de células ganglionares da retina. Por exemplo, volume da escavação é uma medida imprecisa, pois inclui não só a perda da camada de fibras nervosas da retina como também leva em conta o abaulamento posterior da lâmina cribiforme, que não corresponde à medida real da perda de células ganglionares da retina. A análise funcional, que inclui a sensibilidade média, "mean deviation" e valores individuais da sensibilidade ponto a ponto, podem usar uma variedade de testes perimétricos, incluindo a perimetria computadorizada e os novos testes psicofísicos (potencial visual evocado multifocal, "flicker perimetry", perimetria de freqüência dupla) ${ }^{(8)}$. Assim, dependendo do parâmetro estrutural ou anatômico a ser comparado, pode-se encontrar correlações distintas entre a estrutura e a função no glaucoma.

No presente estudo, procurou-se utilizar medidas de avaliação estrutural e funcional usadas na prática clínica diária e observou-se baixa correlação entre elas. Apesar da baixa correlação a avaliação estrutural de Armaly e a escala funcional de Brusini fornecem parâmetros não independentes na avaliação do glaucoma.

\section{ABSTRACT}

Purpose: To evaluate the correlation between Armaly's structural systems with Brusini's functional staging in glaucomatous patients. Methods: Seventy-eight eyes of forty-two patients with primary open-angle glaucoma underwent visual field testing with the Humphrey Field Analyzer II (program SITA 24-2) and optic disc evaluation with the 78D Volk lens by two independent observers. Optic disc was stratified according to cup/disc ratio (C/D), from 0.1 to 1.0. Mean deviation (MD) and pattern standard deviation (PSD) values from each eye were plotted on Brusini's staging system to derive a value for glaucoma severity. Both cup/disc ratio and Brusini's values were plotted on a diagram to assess correlation (Spearman's rho correlation coefficient). Armaly's values were logged in a logarithmic figure and the data plotted on a diagram with Brusini's values for each patient. Results: Armaly's and Brusini's systems dysplayed a poor correlation (rho=0.340). Both unlogged and logged, Armaly's versus Brusini's plots resulted in a linear and weak correlation. Conclusion: Despite the weak correlation, Armaly's structural system and Brusini's staging system provided non-independent measures for glaucoma evaluation.

Keywords: Glaucoma/diagnosis; Glaucoma/classification; Visual fields; Perimetry/methods

\section{REFERÊNCIAS}

1. Betinjane AJ, Paranhos Jr A, Omi CA, Figueiredo CRL, Mandia Júnior C, Silva FA et al. Conceito, fatores de risco e diagnóstico. In: Mello PAA, Mandia Júnior C, organizadores. In: $2^{\circ}$ Consenso Brasileiro de Glaucoma Primário de Ângulo Aberto. São Paulo: Sociedade Brasileira de Glaucoma; 2005. p.4-44.

2. Airaksinen PJ, Drance SM. Neuroretinal rim area and retinal nerve fiber layer in glaucoma. Arch Ophthalmol. 1985;103(2):203-4.

3. Jonas JB, Gründler AE. Correlation between mean visual field loss and morphometric optic disk variables in the open-angle glaucomas. Am J Ophthalmol. 1997;124(4):488-97. Comment in: Am J Ophthalmol. 1997;124(4): 516-20.

4. Garway-Heath DF, Viswanathan A, Westcott M, Kamal D, Fritzke FW, Hitchings RA. Relationship between perimetric light sensitivity and optic disc neuroretinal rim area. In: Wall M, Wild JM, editors. Perimetry update 1998/1999. The Hague: Kugler Publications; 1999. p.381-9.

5. Bartz-Schmidt KU, Thumann G, Jonescu-Cuypers CP, Krieglstein GK. Quantitative morphologic and functional evaluation of the optic nerve head in chronic open-angle glaucoma. Surv Ophthalmol. 1999;44(Suppl 1):S41-53.

6. Garway-Heath DF, Greenfield DS, Caprioli J. Correlation of visual field sensitivity and retinal nerve fibre layer thickness as measured by scanning laser polarimetry [Abstract 980]. In: 2003 Annual Meeting Abstract and Program Planner [monograph on CD ROM]. Bethesda, MD: Association for Research in Vision and Ophthalmology; 2003.

7. Hood DC, Greenstein VC. Multifocal VEP and ganglion cell damage: applications and limitations for the study of glaucoma. Prog Retin Eye Res. 2003;22(2):201-51.

8. Chauhan BC. Comparison of structural and functional methods II. In: Weinreb RN, Greve EL, editors. Glaucoma diagnosis. Structure and function. The Hague: Kugler Publications; 2004. p.145-8.

9. Lemij HG, Reus NJ. Relationship between visual function and retinal nerve fiber layer thickness [Abstract 987]. In: 2003 Annual Meeting Abstract and Program Planner [monography on CD ROM]. Bethesda, MD: Association for Research in Vision and Ophthalmology; 2003.

10. Garway-Heath DF. Comparison of structural and functional methods I. In: Weinreb RN, Greve EL, editors. Glaucoma diagnosis. Structure and function. The Hague: Kugler Publications; 2004. p.135-43.

11. Brusini P. A comparison of three methods for distinguishing between diffuse, localized and mixed visual field defects in glaucoma. In: Wall M, Heijl A, editors. Perimetry update 1996/1997. Amsterdam: Kugler Publications; 1997. p.329-33.

12. Brusini P. Estimating glaucomatous anatomical damage by computerized automated perimetry. Acta Ophthalmol Scand Suppl. 1997;(224):28-9.

13. Armaly MF. The optic cup in the normal eye. I. Cup width, depth, vessel displacement, ocular tension and outflow facility. Am J Ophthalmol. 1969; 68(3):401-7. 